\title{
Improved reachable set bounding for linear time-delay systems with disturbances th $^{2}$
}

\author{
Yin Sheng ${ }^{\mathrm{a}, \mathrm{b}}$, Yi Shen ${ }^{\mathrm{a}, \mathrm{b}, *}$ \\ ${ }^{a}$ School of Automation, Huazhong University of Science and Technology, Wuhan 430074, China \\ ${ }^{b}$ Key Laboratory of Image Processing and Intelligent Control of Education Ministry of China, Wuhan 430074, China
}

\begin{abstract}
In this paper, we reconsider the problem of reachable set bounding for linear time-delay systems with disturbances. Generalized reachable sets for dynamical systems are proposed where initial points are taken into account. Meanwhile, a novel lemma is given which shows an extended criterion that the reachable sets are bounded. Then, based on this lemma and the Lyapunov-Krasovskii functional (LKF) as well as the free-weighting matrix techniques, improved delay-dependent linear matrix inequality (LMI) criteria are obtained for finding an ellipsoid to bound the reachable sets of linear time-delay systems with disturbances. Two numerical examples are finally provided to substantiate the efficiency and merits of our theoretical results.
\end{abstract}

Keywords: reachable set bounding; time-delay systems; Lyapunov-Krasovskii functional; free-weighting matrix

\section{Introduction}

Reachable sets is a set that includes all the state trajectories starting from all allowable initial conditions and inputs. Reachable set bounding is an important and interesting research topic in control theory since it has plenty of applications in control systems with actuator saturation, peak-to-peak gain minimization and aircraft collision avoidance [1, 4]. For example, as indicated in [13], obtaining a smaller ellipsoidal bound of the reachable sets for a linear time-delay system with saturating actuators can allow the designed controller with a larger gain. Hence, over the past few decades, several excellent achievements on reachable set bounding for dynamical systems have been proposed, see e.g., [2-5, 9, 11, 13, 15, 17-19, 21, 26, 27, 29-31].

In [1], Boyd et al. investigated the reachable set bounding for linear systems without any delay by utilizing the quadratic Lyapunov function and S-procedure. This outstanding work motivates the present research. As is well known, time-delay phenomena frequently occur in many real-world systems, and delay-dependent criteria are generally less conservative than delay-independent ones especially when the size of the delay is small $[6-8,12,22-$ $24,28]$. Based on those facts, multiple outcomes on reachable set bounding for time-delay systems have recently been reported [3-5, 11, 13, 15, 19, 21, 26, 27, 29-31], to name a few.

It is worth noting that the results in $[3,4,11,13,19,21,26,29-31]$ are based on Lemma 3 which is proposed in [1]. Yet, in Lemma 3, the initial value $\phi(t)$ of the system is required to be zero, and only the state trajectories from the origin are considered (please refer to Lemma 3 and [1] for details). However, if the initial point $t_{0} \neq 0$, or the value $V\left(x\left(t_{0}\right)\right) \neq 0(V(x(t))$ is the chosen LKF), in these cases, Lemma 3 may not be valid to obtain the ellipsoidal bound for the reachable sets. On the other hand, in [5], based on the Lyapunov-Razumikhin approach, Fridman and Shaked investigated the reachable set estimation for linear time-delay systems with disturbances and LMI criteria were obtained. In [11], Kim considered the reachable set bounding for linear time-delay systems with disturbances

\footnotetext{
This work is supported by the Key Program of National Natural Science Foundation of China (Grant no. 61134012), the National Science Foundation of China (Grant nos. 11271146 and 61374150), and the Prior Development Field for the Doctoral Program of Higher Education of China (Grant no. 20130142130012).

${ }^{*}$ Corresponding author. Tel.: +86 27 87543630; fax: +86 2787543130 .

Email addresses: shengyin90@163.com (Yin Sheng), yishen64@163.com (Yi Shen)
} 
by introducing a modified LKF and using some inequality techniques. Compared with the results in [5], some less

conservative LMI conditions are obtained in [11]. Yet, in [11], $x(t-d)$ and $\int_{t-d}^{t-d(t)} x(s) d s$ are not utilized in the proof $(d(t)$ is the time-varying delay, and $d$ is the upper bound of $d(t))$. Meanwhile, $\mu<1$ is assumed to ensure that the obtained LMIs are feasible (the constant $\mu$ is the upper bound of the derivative $\dot{d}(t)$ ). Hence, there is much room for improvement.

Inspired by the above discussion, in this paper, we shall investigate the reachable set bounding for linear timedelay systems with disturbances where the initial point of the state is considered. To this end, a generalized lemma is firstly given which provides an improved criterion that the reachable sets are bounded on the basis of the initial value $V\left(x\left(t_{0}\right)\right)$. Then, based on this lemma and the LKF as well as free-weighting matrices [8, 25], improved delaydependent LMI conditions are derived for finding an ellipsoid to bound the reachable sets of linear time-delay systems with disturbances. Additionally, the result is extended to the one for linear time-delay systems with both disturbances and polytopic-type uncertainties. Finally, two numerical examples are provided in Section 4 which illustrates that our proposed results are less conservative than some previous ones [11, 13, 19, 30, 31], please see Tables 1 and 2 for more details.

Notations. Throughout this paper, the notations are standard. $\mathbb{R}^{n}$ denotes the $n$-dimensional Euclidean space, and $\mathbb{R}^{n \times m}$ denotes the set of $n \times m$ real matrices. $A>0(A \geq 0)$ means that $A$ is a symmetric positive definite (semidefinite) matrix, meanwhile, $B<0(B \leq 0)$ means that $B$ is a symmetric negative definite (semidefinite) matrix. If $D$ is a vector or matrix, $D^{T}$ represents the transpose of $D . \max \left\{a_{1}, a_{2}, \ldots, a_{n}\right\}\left(\min \left\{a_{1}, a_{2}, \ldots, a_{n}\right\}\right)$ stands for the maximum (minimum) value of $a_{1}, a_{2}, \ldots, a_{n}$. $\mathbb{I}_{\{\mu<1\}}$ is an indicator function which equals to 1 if $\mu<1$ holds, otherwise, if $\mu \geq 1$, then $\mathbb{I}_{\{\mu<1\}}=0$. Symmetric terms in a symmetric matrix are represented by $\star$. In addition, we assume that all the matrices and vectors have compatible dimensions under any algebraic operations.

\section{Preliminaries}

In this paper, we shall consider the following linear time-delay systems with disturbances:

$$
\left\{\begin{array}{l}
\dot{x}(t)=A x(t)+B x(t-d(t))+D w(t) \\
x(t)=\phi(t), \quad t \in[-d, 0]
\end{array}\right.
$$

where $x(t) \in \mathbb{R}^{n}$ is the state vector, and $\phi(t) \in \mathbb{R}^{n}$ is the initial condition. $A, B$ and $D$ are constant matrices with compatible dimensions. $d(t)$ denotes the time-varying delay that satisfies $0 \leq d(t) \leq d$ where $d \geq 0$ is a constant. $w(t) \in \mathbb{R}^{m}$ stands for the disturbance. In order to derive our results, we assume

$$
\dot{d}(t) \leq \mu, \quad w^{T}(t) w(t) \leq \bar{w}^{2},
$$

where $\mu$ and $\bar{w}$ are constants.

Based on $[5,18]$ and the discussion in Section 1, in this paper, we investigate the problem of reachable set bounding for system (1) where the initial point $t_{0} \geq 0$ is considered. The reachable sets of system (1) are denoted by:

$$
\mathbb{U}\left(x, t_{0}, x\left(t_{0}\right)\right) \triangleq\left\{x(t) \in \mathbb{R}^{n} \mid x(t) \text { and } w(t) \text { satisfy (1) and (2), } t \geq t_{0}\right\} .
$$

Define an ellipsoid $\Xi$ which bounds the reachable sets $\mathbb{U}$ of system (1), then

$$
\Xi=\left\{x \in \mathbb{R}^{n}: x^{T} P x \leq 1\right\}
$$

where $P>0$.

In this paper, we try to obtain the delay-dependent LMI conditions for finding an ellipsoid $\Xi$ defined in (4) as small as possible that bounds the reachable sets $\mathbb{U}$ defined in (3) of system (1).

Before moving on, some lemmas are given which have important roles in our proofs.

Lemma 1. [14] For any symmetric positive definite matrix $H \in \mathbb{R}^{n \times n}$ and a scalar $h>0$ such that the following integrations are well-defined, then

$$
h \int_{t-h}^{t} x^{T}(s) H x(s) d s \geq\left[\int_{t-h}^{t} x(s) d s\right]^{T} H\left[\int_{t-h}^{t} x(s) d s\right] .
$$


Lemma 2. [20] Let $\psi_{1}, \psi_{2}, \ldots, \psi_{n}: \mathbb{R}^{m} \rightarrow \mathbb{R}$ have positive values in an open subset $\Omega$ of $\mathbb{R}^{m}$. Then, the reciprocally convex combination of $\psi_{i}$ over $\Omega$ satisfies

$$
\min _{\left\{\kappa_{i} \mid \kappa_{i}>0, \sum_{i} \kappa_{i}=1\right\}} \sum_{i} \frac{1}{\kappa_{i}} \psi_{i}(t)=\sum_{i} \psi_{i}(t)+\max _{\varphi_{i, j}(t)} \sum_{i \neq j} \varphi_{i, j}(t)
$$

subject to

$$
\left\{\varphi_{i, j}(t): \mathbb{R}^{m} \mapsto \mathbb{R}, \varphi_{j, i}(t)=\varphi_{i, j}(t),\left[\begin{array}{cc}
\psi_{i}(t) & \varphi_{i, j}(t) \\
\varphi_{j, i}(t) & \psi_{j}(t)
\end{array}\right] \geq 0\right\} .
$$

Lemma 3. [1] Let $V(x(t))$ be a Lyapunov function for system (1), $V(x(0))=0$ and $\phi(t)=0$ for $t \in[-d, 0]$. If $\dot{V}(x(t))+\alpha V(x(t))-\frac{\alpha}{\bar{w}^{2}} w^{T}(t) w(t) \leq 0$ with a scalar $\alpha>0$, and $\bar{w}$ is defined in (2), then $V(x(T)) \leq 1$ for $T \geq 0$.

Lemma 4. Let $V(x(t))$ be a Lyapunov function for system (1), $V\left(x\left(t_{0}\right)\right) \leq \frac{\beta \bar{w}^{2}}{\alpha}, t_{0} \geq 0, \alpha>0, \beta>0$, the constant $\bar{w}$ is defined in (2). If $\dot{V}(x(t))+\alpha V(x(t))-\beta w^{T}(t) w(t) \leq 0$, then $V(x(t)) \leq \frac{\beta \bar{w}^{2}}{\alpha}$ for $t \geq t_{0}$.

Proof. By (2), $\alpha>0, \beta>0$ and $\dot{V}(x(t))+\alpha V(x(t))-\beta w^{T}(t) w(t) \leq 0$, we have

$$
\dot{V}(x(t))+\alpha V(x(t)) \leq \beta \bar{w}^{2} .
$$

It is clear that (8) is equivalent to

$$
\frac{\mathrm{d}\left[V(x(t))-\frac{\beta \bar{w}^{2}}{\alpha}\right]}{\mathrm{d} t} \leq-\alpha\left[V(x(t))-\frac{\beta \bar{w}^{2}}{\alpha}\right] .
$$

Let

$$
U(t) \triangleq V(x(t))-\frac{\beta \bar{w}^{2}}{\alpha}
$$

then (9) is transformed into

$$
\frac{\mathrm{d} U(t)}{\mathrm{d} t} \leq-\alpha U(t)
$$

Let us consider $e^{\alpha t} U(t)$, then we obtain

$$
\frac{\mathrm{d}\left[e^{\alpha t} U(t)\right]}{\mathrm{d} t}=e^{\alpha t}[\dot{U}(t)+\alpha U(t)] \leq 0
$$

since $\dot{U}(t)+\alpha U(t) \leq 0$ according to (11). Then, integrating (12) from $t_{0}$ to $t \geq t_{0}$ yields

$$
e^{\alpha t} U(t)-e^{\alpha t_{0}} U\left(t_{0}\right) \leq 0,
$$

which means

$$
U(t) \leq U\left(t_{0}\right) \exp \left\{-\alpha\left(t-t_{0}\right)\right\}
$$

Substituting $U(t)=V(x(t))-\frac{\beta \bar{w}^{2}}{\alpha}$ into (14) gives:

$$
V(x(t))-\frac{\beta \bar{w}^{2}}{\alpha} \leq\left[V\left(x\left(t_{0}\right)\right)-\frac{\beta \bar{w}^{2}}{\alpha}\right] \exp \left\{-\alpha\left(t-t_{0}\right)\right\}, \quad t \geq t_{0} .
$$

Now we investigate (15), since $\alpha>0, \beta>0$ and $\exp \left\{-\alpha\left(t-t_{0}\right)\right\} \geq 0$, if $V\left(x\left(t_{0}\right)\right) \leq \frac{\beta \bar{w}^{2}}{\alpha}$, then it is obvious that $V(x(t))-\frac{\beta \bar{w}^{2}}{\alpha} \leq 0$, namely, $V(x(t)) \leq \frac{\beta \bar{w}^{2}}{\alpha}$ holds for any $t>t_{0}$. In summary, for any initial point $t_{0} \geq 0$, if $V\left(x\left(t_{0}\right)\right) \leq \frac{\beta \bar{w}^{2}}{\alpha}$, then $V(x(t))$ will not exceed $\frac{\beta \bar{w}^{2}}{\alpha}$ for $t>t_{0}$. The proof is complete. 
Remark 1. It should be pointed out, if we set $\beta=\frac{\alpha}{\bar{w}^{2}}, t_{0}=0, V(x(0))=0$ and $\phi(t)=0$ for $t \in[-d, 0]$, then Lemma 4 reduces to Lemma 3. By contrast, Lemma 4 provides a more general result for the problem of reachable set bounding of system (1). As shown in $[3,4,11,13,19,21,26,29-31]$, the scholars investigate the reachable set bounding under the assumptions that the initial conditions $\phi(t)$ of the systems are zero and state trajectories start from the origin. However, in Lemma 4, we illustrate that for any initial point $t_{0} \geq 0$, the reachable sets $\mathbb{U}$ defined in (3) of system (1) can be bounded if $V\left(x\left(t_{0}\right)\right) \leq \frac{\beta \bar{w}^{2}}{\alpha}$ holds.

\section{Main Results}

In this section, we will present our main results. Improved delay-dependent LMI criteria are proposed for finding an ellipsoid $\Xi$ defined in (4) to bound the reachable sets $\mathbb{U}$ defined in (3) of system (1). For simplicity, in the following part, we will utilize the notation $V(t)$ instead of $V(x(t))$.

Theorem 1. For given scalars $d \geq 0, \mu$ and $\bar{w}$, if there exist appropriately dimensioned matrices $P>0, Q>0, R>$ $0, M>0, N>0$, symmetric matrices $X, Z$, any matrices $Y, E, E_{1}, F^{T}=\left[F_{1}^{T}, F_{2}^{T}, \ldots, F_{7}^{T}\right]$ and a scalar $\alpha>0$ such that the following LMIs hold:

$$
\begin{aligned}
& \Gamma=\left[\begin{array}{ccccccc}
\Gamma_{11} & \Gamma_{12} & \Gamma_{13} & \Gamma_{14} & \Gamma_{15} & \Gamma_{16} & -F_{1}+A^{T} F_{7}^{T} \\
\star & \Gamma_{22} & \Gamma_{23} & \Gamma_{24} & \Gamma_{25} & F_{2} D+B^{T} F_{6}^{T} & -F_{2}+B^{T} F_{7}^{T} \\
\star & \star & -e^{-\alpha d}(R+N) & -Z & -Z & F_{3} D & -F_{3} \\
\star & \star & \star & \Gamma_{44} & \Gamma_{45} & Y^{T} D+F_{4} D & -F_{4} \\
\star & \star & \star & \star & \Gamma_{55} & Y^{T} D+F_{5} D & -F_{5} \\
\star & \star & \star & \star & \star & \Gamma_{66} & -F_{6}+D^{T} F_{7}^{T} \\
\star & \star & \star & \star & \star & \star & \Gamma_{77}
\end{array}\right] \leq 0, \\
& \left(\begin{array}{cc}
X & Y \\
\star & Z
\end{array}\right)>0,\left(\begin{array}{cc}
M & E \\
\star & M
\end{array}\right) \geq 0,\left(\begin{array}{cc}
N & E_{1} \\
\star & N
\end{array}\right) \geq 0,
\end{aligned}
$$

where

$$
\begin{aligned}
\Gamma_{11}= & P A+A^{T} P+\alpha P+Q+R+d M+X A+A^{T} X \\
& +Y+Y^{T}+\alpha X-e^{-\alpha d} N+F_{1} A+A^{T} F_{1}^{T}, \\
\Gamma_{12}= & P B+X B+e^{-\alpha d}\left(N-E_{1}\right)+F_{1} B+A^{T} F_{2}^{T}, \\
\Gamma_{13}= & -Y+e^{-\alpha d} E_{1}+A^{T} F_{3}^{T}, \\
\Gamma_{14}= & A^{T} Y+\alpha Y+Z+A^{T} F_{4}^{T}, \\
\Gamma_{15}= & A^{T} Y+\alpha Y+Z+A^{T} F_{5}^{T}, \\
\Gamma_{16}= & P D+X D+A^{T} F_{6}^{T}+F_{1} D, \\
\Gamma_{22}= & e^{-\alpha d}\left[(\mu-1) Q \mathbb{I}_{\{\mu<1\}}+E_{1}+E_{1}^{T}-2 N\right]+(\mu-1) Q \mathbb{I}_{\{\mu \geq 1\}}+F_{2} B+B^{T} F_{2}^{T}, \\
\Gamma_{23}= & e^{-\alpha d}\left(N-E_{1}\right)+B^{T} F_{3}^{T}, \\
\Gamma_{24}= & B^{T} Y+B^{T} F_{4}^{T}, \\
\Gamma_{25}= & B^{T} Y+B^{T} F_{5}^{T}, \\
\Gamma_{44}= & -\frac{e^{-\alpha d}}{d} M+\alpha Z, \\
\Gamma_{45}= & -\frac{e^{-\alpha d}}{d} E+\alpha Z, \\
\Gamma_{55}= & -\frac{e^{-\alpha d}}{d} M+\alpha Z, \\
\Gamma_{66}= & F_{6} D+D^{T} F_{6}^{T}-\frac{\alpha}{\bar{w}^{2}}, \\
\Gamma_{77}= & d^{2} N-F_{7}-F_{7}^{T},
\end{aligned}
$$


in which $\mathbb{I}_{\{\mu<1\}}$ and $\mathbb{I}_{\{\mu \geq 1\}}$ are indicator functions, then, the reachable sets $\mathbb{U}$ defined in (3) of system (1) can be bounded by the ellipsoid $\Xi$ defined in (4).

Proof. To prove this theorem, let us consider the following LKF candidate:

$$
V(t)=\sum_{i=1}^{6} V_{i}(t)
$$

where

$$
\begin{aligned}
& V_{1}(t)=x^{T}(t) P x(t), \\
& V_{2}(t)=\int_{t-d(t)}^{t} e^{\alpha(s-t)} x^{T}(s) Q x(s) d s, \\
& V_{3}(t)=\int_{t-d}^{t} e^{\alpha(s-t)} x^{T}(s) R x(s) d s, \\
& V_{4}(t)=\int_{t-d}^{t} e^{\alpha(s-t)}(d-t+s) x^{T}(s) M x(s) d s, \\
& V_{5}(t)=\left[\begin{array}{cc}
\int_{t-d}^{t} x(t) \\
x(s) d s
\end{array}\right]^{T}\left[\begin{array}{cc}
X & Y \\
\star & Z
\end{array}\right]\left[\begin{array}{c}
x(t) \\
\int_{t-d}^{t} x(s) d s
\end{array}\right], \\
& V_{6}(t)=d \int_{-d}^{0} \int_{t+\theta}^{t} e^{\alpha(s-t)} \dot{x}^{T}(s) N \dot{x}(s) d s d \theta .
\end{aligned}
$$

Calculating the time derivative of $V(t)$ along the trajectory of system (1) and combining the condition (2) give

$$
\begin{aligned}
& \dot{V}_{1}(t)=2 x^{T}(t) P \dot{x}(t)-\alpha V_{1}(t)+\alpha V_{1}(t) \\
& =2 x^{T}(t) P[A x(t)+B x(t-d(t))+D w(t)]-\alpha V_{1}(t)+\alpha x^{T}(t) P x(t), \\
& \dot{V}_{2}(t)=-\alpha V_{2}(t)+x^{T}(t) Q x(t)-[1-\dot{d}(t)] e^{-\alpha d(t)} x^{T}(t-d(t)) Q x(t-d(t)) \\
& \leq-\alpha V_{2}(t)+x^{T}(t) Q x(t)-(1-\mu) e^{-\alpha d(t)} x^{T}(t-d(t)) Q x(t-d(t)) \\
& \leq-\alpha V_{2}(t)+x^{T}(t) Q x(t)-(1-\mu) e^{-\alpha d} x^{T}(t-d(t)) Q x(t-d(t)) \mathbb{I}_{\{\mu<1\}} \\
& -(1-\mu) x^{T}(t-d(t)) Q x(t-d(t)) \mathbb{I}_{\{\mu \geq 1\}}, \\
& \dot{V}_{3}(t)=-\alpha V_{3}(t)+x^{T}(t) R x(t)-e^{-\alpha d} x^{T}(t-d) R x(t-d), \\
& \dot{V}_{4}(t)=-\alpha V_{4}(t)+d x^{T}(t) M x(t)-\int_{t-d}^{t} e^{\alpha(s-t)} x^{T}(s) M x(s) d s, \\
& \dot{V}_{5}(t)=-\alpha V_{5}(t)+2\left[\begin{array}{c}
x(t) \\
\int_{t-d}^{t} x(s) d s
\end{array}\right]^{T}\left[\begin{array}{ll}
X & Y \\
\star & Z
\end{array}\right]\left[\begin{array}{c}
\dot{x}(t) \\
x(t)-x(t-d)
\end{array}\right] \\
& +\alpha\left[\begin{array}{c}
x(t) \\
\int_{t-d}^{t} x(s) d s
\end{array}\right]^{T}\left[\begin{array}{cc}
X & Y \\
\star & Z
\end{array}\right]\left[\begin{array}{c}
x(t) \\
\int_{t-d}^{t} x(s) d s
\end{array}\right] \\
& =-\alpha V_{5}(t)+2\left[x^{T}(t) X+\int_{t-d}^{t} x^{T}(s) d s Y^{T}\right][A x(t)+B x(t-d(t))+D w(t)] \\
& +2\left[x^{T}(t) Y+\int_{t-d}^{t} x^{T}(s) d s Z\right][x(t)-x(t-d)] \\
& +\alpha x^{T}(t) P x(t)+2 \alpha x^{T}(t) Y \int_{t-d}^{t} x(s) d s+\alpha \int_{t-d}^{t} x^{T}(s) d s Z \int_{t-d}^{t} x(s) d s, \\
& \dot{V}_{6}(t)=-\alpha V_{6}(t)+d^{2} \dot{x}^{T}(t) N \dot{x}(t)-d \int_{t-d}^{t} e^{\alpha(s-t)} \dot{x}^{T}(s) N \dot{x}(s) d s .
\end{aligned}
$$


Now we deal with the integration $-\int_{t-d}^{t} e^{\alpha(s-t)} x^{T}(s) M x(s) d s$ in (43), by Lemma 1, Lemma 2 and (17), we have

$$
\begin{aligned}
& -\int_{t-d}^{t} e^{\alpha(s-t)} x^{T}(s) M x(s) d s \\
& \leq-e^{-\alpha d} \int_{t-d}^{t} x^{T}(s) M x(s) d s \\
& =-e^{-\alpha d}\left[\int_{t-d(t)}^{t} x^{T}(s) M x(s) d s+\int_{t-d}^{t-d(t)} x^{T}(s) M x(s) d s\right] \\
& =-\frac{e^{-\alpha d}}{d} \frac{d}{d(t)} d(t) \int_{t-d(t)}^{t} x^{T}(s) M x(s) d s \\
& -\frac{e^{-\alpha d}}{d} \frac{d}{d-d(t)}(d-d(t)) \int_{t-d}^{t-d(t)} x^{T}(s) M x(s) d s \\
& \leq-\frac{e^{-\alpha d}}{d} \frac{d}{d(t)}\left[\int_{t-d(t)}^{t} x(s) d s\right]^{T} M\left[\int_{t-d(t)}^{t} x(s) d s\right] \\
& -\frac{e^{-\alpha d}}{d} \frac{d}{d-d(t)}\left[\int_{t-d}^{t-d(t)} x(s) d s\right]^{T} M\left[\int_{t-d}^{t-d(t)} x(s) d s\right] \\
& \leq-\frac{e^{-\alpha d}}{d}\left[\begin{array}{l}
\int_{t-d(t)}^{t} x(s) d s \\
\int_{t-d}^{t-d(t)} x(s) d s
\end{array}\right]^{T}\left[\begin{array}{cc}
M & E \\
\star & M
\end{array}\right]\left[\begin{array}{l}
\int_{t-d(t)}^{t} x(s) d s \\
\int_{t-d}^{t-d(t)} x(s) d s
\end{array}\right]
\end{aligned}
$$

where the matrices $M$ and $E$ satisfy $\left[\begin{array}{cc}M & E \\ \star & M\end{array}\right] \geq 0$.

For the integration $-d \int_{t-d}^{t} e^{\alpha(s-t)} \dot{x}^{T}(s) N \dot{x}(s) d s$ in (45), similar to the methods in (46), one has

$$
\begin{aligned}
& -d \int_{t-d}^{t} e^{\alpha(s-t)} \dot{x}^{T}(s) N \dot{x}(s) d s \\
\leq & -e^{-\alpha d}\left[\begin{array}{l}
\int_{t-d(t)}^{t} \dot{x}(s) d s \\
\int_{t-d}^{t-d(t)} \dot{x}(s) d s
\end{array}\right]^{T}\left[\begin{array}{cc}
N & E_{1} \\
\star & N
\end{array}\right]\left[\begin{array}{c}
\int_{t-d(t)}^{t} \dot{x}(s) d s \\
\int_{t-d}^{t-d(t)} \dot{x}(s) d s
\end{array}\right] \\
\leq & -e^{-\alpha d}\left[\begin{array}{c}
x(t)-x(t-d(t)) \\
x(t-d(t))-x(t-d)
\end{array}\right]^{T}\left[\begin{array}{cc}
N & E_{1} \\
\star & N
\end{array}\right]\left[\begin{array}{c}
x(t)-x(t-d(t)) \\
x(t-d(t))-x(t-d)
\end{array}\right]
\end{aligned}
$$

where the matrices $N$ and $E_{1}$ satisfy $\left[\begin{array}{cc}N & E_{1} \\ \star & N\end{array}\right] \geq 0$.

On the other hand, based on system (1), for any matrices $F^{T}=\left[F_{1}^{T}, F_{2}^{T}, \ldots, F_{7}^{T}\right]$ with appropriate dimensions, the following equality is true:

$$
2 \xi^{T}(t) F[-\dot{x}(t)+A x(t)+B x(t-d(t))+D w(t)]=0
$$

where

$$
\xi^{T}(t)=\left\{x^{T}(t), x^{T}(t-d(t)), x^{T}(t-d), \int_{t-d(t)}^{t} x^{T}(s) d s, \int_{t-d}^{t-d(t)} x^{T}(s) d s, w^{T}(t), \dot{x}^{T}(t)\right\} .
$$

Then, substituting (46) and (47) into (43) and (45), respectively, and combining (40)-(42), (44), (48), we have

$$
\dot{V}(t)+\alpha V(t)-\frac{\alpha}{\bar{w}^{2}} w^{T}(t) w(t) \leq \xi^{T}(t) \Gamma \xi(t) \leq 0,
$$

where $\Gamma$ and $\xi(t)$ are defined in (16) and (49), respectively. By (50) and Lemma 4, for any initial point $t_{0} \geq 0$, if $V\left(t_{0}\right) \leq 1$, then $V(t) \leq 1$ for any $t \geq t_{0}$. Based on the definition of the LKF in (33), we therefore have $x^{T}(t) P x(t) \leq$ $V(t) \leq 1$, which means that the reachable sets $\mathbb{U}$ defined in (3) of system (1) can be bounded by the ellipsoid $\Xi$ defined in (4). This completes the proof. 
Remark 2. It should be noted that compared with the results in $[1,4,5,11,13,19,31]$, our system is more common since in system (1), the initial condition $\phi(t)$ is not required to be zero, and our result is more general since we derive an improved criterion that the reachable sets $\mathbb{U}$ defined in (3) of system (1) are bounded by the ellipsoid $\Xi$ defined in (4) on the basis of the value $V\left(x\left(t_{0}\right)\right)$. On the other hand, our result is less conservative than the ones in [11, 13, 19,30,31], please see Tables 1 and 2 for more details.

Remark 3. In [[11], Th. 1], $\dot{d}(t) \leq \mu<1$ is required to ensure the rationality of the enlargement of $-[1-$ $\dot{d}(t)] e^{-\alpha d(t)} x^{T}(t-d(t)) S x(t-d(t))$ and the feasibility of the proposed LMI criteria. In our paper, to break through these restrictions, we introduce two indicator functions to solve the enlargement of $-[1-\dot{d}(t)] e^{-\alpha d(t)} x^{T}(t-d(t)) Q x(t-d(t))$ in (41), meanwhile, by employing the free-weighting matrix $F$, LMI (16) is feasible even if $\mu \geq 1$.

Remark 4. For system (1), when $w(t)=0$, namely, there is no disturbance in system (1). In this case, system (1) reduces to the nominal time-delay system $\dot{x}(t)=A x(t)+B x(t-d(t))$. Based on the Lyapunov stability theory $[10,16]$ and the analysis in Lemma 4, Theorem 1 provides an exponential stability result for the nominal time-delay system $\dot{x}(t)=A x(t)+B x(t-d(t))$. Due to space limitations, corresponding result is omitted here.

To find the ellipsoid $\Xi$ as small as possible, we use the same approach that is proposed in [5]:

maximize $\delta$ subject to $\delta I \leq P$ and this is equivalent to minimize $\bar{\delta}$ subject to

$$
\left(\begin{array}{cc}
\bar{\delta} I & I \\
I & P
\end{array}\right) \geq 0
$$

with $\bar{\delta}=1 / \delta$.

Now we turn to consider the following time-delay systems with disturbances and polytopic-type uncertainties:

$$
\dot{x}(t)=\left(A_{0}+\Delta A(t)\right) x(t)+\left(B_{0}+\Delta B(t)\right) x(t-d(t))+\left(D_{0}+\Delta D(t)\right) w(t)
$$

where $\Delta A(t)=\sum_{i=1}^{N} \rho_{i}(t) A_{i}, \Delta B(t)=\sum_{i=1}^{N} \rho_{i}(t) B_{i}$ and $\Delta D(t)=\sum_{i=1}^{N} \rho_{i}(t) D_{i}$, in which $0 \leq \rho_{i}(t) \leq 1$ and $\sum_{i=1}^{N} \rho_{i}(t)=1$, $\forall t \geq-d$. Then we have following corollary.

Corollary 1. For given scalars $d \geq 0, \mu$ and $\bar{w}$, if there exist appropriately dimensioned matrices $\tilde{P}>0, \tilde{Q}>0, \tilde{R}>$ $0, \tilde{M}>0, \tilde{N}>0$, symmetric matrices $\tilde{X}, \tilde{Z}$, any matrices $\tilde{Y}, \tilde{E}, \tilde{E}_{1}, \tilde{F}^{T}=\left[\tilde{F}_{1}^{T}, \tilde{F}_{2}^{T}, \ldots, \tilde{F}_{7}^{T}\right]$ and a scalar $\tilde{\alpha}>0$ such that the following LMIs hold:

$$
\begin{aligned}
& \tilde{\Gamma}= {\left[\begin{array}{ccccccc}
\tilde{\Gamma}_{11} & \tilde{\Gamma}_{12} & \tilde{\Gamma}_{13} & \tilde{\Gamma}_{14} & \tilde{\Gamma}_{15} & \tilde{\Gamma}_{16} & \tilde{\Gamma}_{17} \\
\star & \tilde{\Gamma}_{22} & \tilde{\Gamma}_{23} & \tilde{\Gamma}_{24} & \tilde{\Gamma}_{25} & \tilde{\Gamma}_{26} & \tilde{\Gamma}_{27} \\
\star & \star & -e^{-\tilde{\alpha} d}(\tilde{R}+\tilde{N}) & -\tilde{Z} & -\tilde{Z} & \tilde{F}_{3}\left(D_{0}+D_{i}\right) & -\tilde{F}_{3} \\
\star & \star & \star & \tilde{\Gamma}_{44} & \tilde{\Gamma}_{45} & \tilde{\Gamma}_{46} & -\tilde{F}_{4} \\
\star & \star & \star & \star & \tilde{\Gamma}_{44} & \tilde{\Gamma}_{56} & -\tilde{F}_{5} \\
\star & \star & \star & \star & \star & \tilde{\Gamma}_{66} & \tilde{\Gamma}_{67} \\
\star & \star & \star & \star & \star & \star & d^{2} \tilde{N}-\tilde{F}_{7}-\tilde{F}_{7}^{T}
\end{array}\right] \leq 0, i=1,2, \ldots, N, } \\
&\left(\begin{array}{cc}
\tilde{X} & \tilde{Y} \\
\star & \tilde{Z}
\end{array}\right)>0,\left(\begin{array}{cc}
\tilde{M} & \tilde{E} \\
\star & \tilde{M}
\end{array}\right) \geq 0,\left(\begin{array}{cc}
\tilde{N} & \tilde{E}_{1} \\
\star & \tilde{N}
\end{array}\right) \geq 0,
\end{aligned}
$$

where

$$
\begin{aligned}
\tilde{\Gamma}_{11}= & \tilde{P}\left(A_{0}+A_{i}\right)+\left(A_{0}+A_{i}\right)^{T} \tilde{P}+\tilde{\alpha} \tilde{P}+\tilde{Q}+\tilde{R}+d \tilde{M} \\
& +\tilde{X}\left(A_{0}+A_{i}\right)+\left(A_{0}+A_{i}\right)^{T} \tilde{X}+\tilde{Y}+\tilde{Y}^{T}+\tilde{\alpha} \tilde{X} \\
& -e^{-\tilde{\alpha} d} \tilde{N}+\tilde{F}_{1}\left(A_{0}+A_{i}\right)+\left(A_{0}+A_{i}\right)^{T} \tilde{F}_{1}^{T}, \\
\tilde{\Gamma}_{12}= & (\tilde{P}+\tilde{X})\left(B_{0}+B_{i}\right)+e^{-\tilde{\alpha} d}\left(\tilde{N}-\tilde{E}_{1}\right)+\tilde{F}_{1}\left(B_{0}+B_{i}\right)+\left(A_{0}+A_{i}\right)^{T} \tilde{F}_{2}^{T}, \\
\tilde{\Gamma}_{13}= & e^{-\tilde{\alpha} d} \tilde{E}_{1}-\tilde{Y}+\left(A_{0}+A_{i}\right)^{T} \tilde{F}_{3}^{T}, \\
\tilde{\Gamma}_{14}= & \left(A_{0}+A_{i}\right)^{T} \tilde{Y}+\tilde{\alpha} \tilde{Y}+\tilde{Z}+\left(A_{0}+A_{i}\right)^{T} \tilde{F}_{4}^{T},
\end{aligned}
$$


Table 1: Comparison of $\bar{\delta}$ for various $d$

\begin{tabular}{c|ccccc}
\hline$d$ & 0.1 & 0.3 & 0.5 & 0.7 & 0.9 \\
\hline Theorem 1 & 0.57 & 0.62 & 0.88 & 1.61 & 3.13 \\
{$[11]$} & 0.83 & 1.28 & 1.94 & 2.90 & 4.46 \\
{$[13]$} & 0.68 & 0.80 & 0.97 & 1.64 & 3.22 \\
{$[19]$} & 0.74 & 0.92 & 1.36 & 2.30 & 3.51 \\
{$[30]$} & 0.66 & 0.75 & 0.94 & 1.61 & 3.14 \\
{$[31]$} & 0.66 & 0.75 & 0.94 & 1.61 & 3.14 \\
\hline
\end{tabular}

$$
\begin{aligned}
\tilde{\Gamma}_{15}= & \left(A_{0}+A_{i}\right)^{T} \tilde{Y}+\tilde{\alpha} \tilde{Y}+\tilde{Z}+\left(A_{0}+A_{i}\right)^{T} \tilde{F}_{5}^{T}, \\
\tilde{\Gamma}_{16}= & \left(\tilde{P}+\tilde{X}+\tilde{F}_{1}\right)\left(D_{0}+D_{i}\right)+\left(A_{0}+A_{i}\right)^{T} \tilde{F}_{6}^{T}, \\
\tilde{\Gamma}_{17}= & -\tilde{F}_{1}+\left(A_{0}+A_{i}\right)^{T} \tilde{F}_{7}^{T}, \\
\tilde{\Gamma}_{22}= & e^{-\tilde{\alpha} d}\left[(\mu-1) \tilde{Q} \mathbb{I}_{\{\mu<1\}}+\tilde{E}_{1}+\tilde{E}_{1}^{T}-2 \tilde{N}\right] \\
& +(\mu-1) \tilde{Q} \mathbb{I}_{\{\mu \geq 1\}}+\tilde{F}_{2}\left(B_{0}+B_{i}\right)+\left(B_{0}+B_{i}\right)^{T} \tilde{F}_{2}^{T}, \\
\tilde{\Gamma}_{23}= & e^{-\tilde{\alpha} d}\left(\tilde{N}-\tilde{E}_{1}\right)+\left(B_{0}+B_{i}\right)^{T} \tilde{F}_{3}^{T}, \\
\tilde{\Gamma}_{24}= & \left(B_{0}+B_{i}\right)^{T} \tilde{Y}+\left(B_{0}+B_{i}\right)^{T} \tilde{F}_{4}^{T}, \\
\tilde{\Gamma}_{25}= & \left(B_{0}+B_{i}\right)^{T} \tilde{Y}+\left(B_{0}+B_{i}\right)^{T} \tilde{F}_{5}^{T}, \\
\tilde{\Gamma}_{26}= & \tilde{F}_{2}\left(D_{0}+D_{i}\right)+\left(B_{0}+B_{i}\right)^{T} \tilde{F}_{6}^{T}, \\
\tilde{\Gamma}_{27}= & -\tilde{F}_{2}+\left(B_{0}+B_{i}\right)^{T} \tilde{F}_{7}^{T}, \\
\tilde{\Gamma}_{44}= & -\frac{e^{-\tilde{\alpha} d}}{d} \tilde{M}+\tilde{\alpha} \tilde{Z}, \\
\tilde{\Gamma}_{45}= & -\frac{e^{-\tilde{\alpha} d}}{d} \tilde{E}+\tilde{\alpha} \tilde{Z}, \\
\tilde{\Gamma}_{46}= & \tilde{Y}^{T}\left(D_{0}+D_{i}\right)+\tilde{F}_{4}\left(D_{0}+D_{i}\right), \\
\tilde{\Gamma}_{56}= & \tilde{Y}^{T}\left(D_{0}+D_{i}\right)+\tilde{F}_{5}\left(D_{0}+D_{i}\right), \\
\tilde{\Gamma}_{66}= & \tilde{F}_{6}\left(D_{0}+D_{i}\right)+\left(D_{0}+D_{i}\right)^{T} \tilde{F}_{6}^{T}-\frac{\tilde{\alpha}}{\bar{w}^{2}}, \\
\tilde{\Gamma}_{67}= & -\tilde{F}_{6}+\left(D_{0}+D_{i}\right)^{T} \tilde{F}_{7}^{T},
\end{aligned}
$$

in which $\mathbb{I}_{\{\mu<1\}}$ and $\mathbb{I}_{\{\mu \geq 1\}}$ are indicator functions, then, the reachable sets of system (52) can be bounded by the ellipsoid $\Xi$ defined in (4).

Proof. This corollary is a direct extension of Theorem 1, so the proof is omitted here.

\section{Numerical Example}

In this section, two numerical examples are provided to demonstrate the feasibility and values of Theorem 1 and Corollary 1.

Example 1. Consider system (1) with the following coefficients:

$$
A=\left[\begin{array}{cc}
-2 & 0 \\
0 & -0.7
\end{array}\right], B=\left[\begin{array}{cc}
-1 & 0 \\
-1 & -0.9
\end{array}\right], D=\left[\begin{array}{c}
-0.5 \\
1
\end{array}\right],
$$

and $\mu=0, \bar{w}=1$. By using the result in Theorem 1, we can calculate the values $\bar{\delta}$ for different $d$ by LMI toolbox in MATLAB R2014a. Corresponding results are explicitly listed in Table 1. According to Table 1, it is clear that the values $\bar{\delta}$ that are computed by Theorem 1 are smaller than the results in $[11,13,19,30,31]$. Meanwhile, as to different 


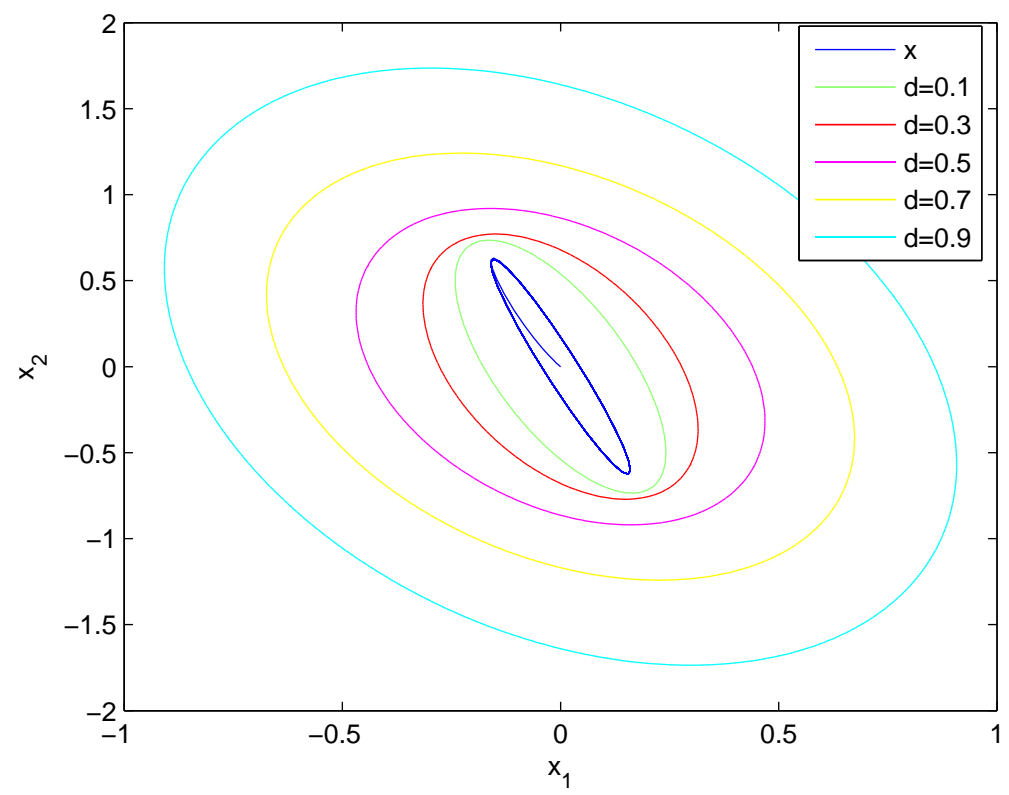

Figure 1: The reachable sets and ellipsoidal bounds of Example 1

$d$, corresponding ellipsoidal bounds of the reachable sets for Example 1 are depicted in Fig. 1 which indicates that the reachable sets of system (1) can be bounded by the ellipsoid $\Xi$. On the other hand, based on the analysis in Lemma 4 and the construction of $V(t)$, for any initial point $t_{0} \geq 0$, if

$$
\begin{aligned}
V\left(t_{0}\right)= & x^{T}\left(t_{0}\right) P x\left(t_{0}\right)+\int_{t_{0}-d\left(t_{0}\right)}^{t_{0}} e^{\alpha\left(s-t_{0}\right)} x^{T}(s) Q x(s) d s+\int_{t_{0}-d}^{t_{0}} e^{\alpha\left(s-t_{0}\right)} x^{T}(s) R x(s) d s \\
& +\int_{t_{0}-d}^{t_{0}} e^{\alpha\left(s-t_{0}\right)}\left(d-t_{0}+s\right) x^{T}(s) M x(s) d s+\left[\begin{array}{c}
x\left(t_{0}\right) \\
\int_{t_{0}-d}^{t_{0}} x(s) d s
\end{array}\right]^{T}\left[\begin{array}{cc}
X & Y \\
\star & Z
\end{array}\right]\left[\begin{array}{c}
x\left(t_{0}\right) \\
\int_{t_{0}-d}^{t_{0}} x(s) d s
\end{array}\right] \\
& +d \int_{-d}^{0} \int_{t_{0}+\theta}^{t_{0}} e^{\alpha\left(s-t_{0}\right)} \dot{x}^{T}(s) N \dot{x}(s) d s d \theta \\
\leq & 1,
\end{aligned}
$$

then the state trajectories starting from $t_{0}$ of system (1) can be bounded by the ellipsoid $\Xi$.

Example 2. Consider system (52) with following parameters [11, 31]:

$$
\begin{aligned}
& A_{0}=\left[\begin{array}{cc}
-2 & 0 \\
0 & -0.9
\end{array}\right], B_{0}=\left[\begin{array}{cc}
-1 & 0 \\
-1 & -1
\end{array}\right], D_{0}=\left[\begin{array}{c}
-0.5 \\
1
\end{array}\right], A_{1}=\left[\begin{array}{cc}
0 & 0 \\
0 & 0.2
\end{array}\right], \\
& A_{2}=\left[\begin{array}{cc}
0 & 0 \\
0 & -0.2
\end{array}\right], B_{1}=\left[\begin{array}{cc}
0 & 0 \\
0 & 0.1
\end{array}\right], B_{2}=\left[\begin{array}{cc}
0 & 0 \\
0 & -0.1
\end{array}\right], \bar{w}=1 .
\end{aligned}
$$

Using Corollary 1, we can also calculate the value $\bar{\delta}$. In Table 2, we calculate the values $\bar{\delta}$ with various $\mu, d=0.75$. The results in $[11,31]$ are also listed in Table 2. Note that the results in [5] are independent with the parameter $\mu$, and when $d=0.75$, the value $\bar{\delta}$ is 65.42 . Therefore, it is clear that our results are less conservative than the ones in $[5,11,31]$.

\section{Conclusions}

In this paper, we have investigated the reachable set bounding for linear time-delay systems with disturbances. By using the LKF and free-weighting matrices, improved delay-dependent LMI conditions were built for finding 
Table 2: Comparison of $\bar{\delta}$ for various $\mu$ with $d=0.75$

\begin{tabular}{c|ccc}
\hline$\mu$ & 0.0 & 0.5 & 2 \\
\hline Corollary 1 & 2.27 & 2.29 & 2.29 \\
\hline$[11]$ & 3.34 & 18.36 & - \\
\hline$[31]$ & 2.28 & 2.85 & 13.39 \\
\hline
\end{tabular}

an ellipsoid to bound the reachable sets of linear time-delay systems with disturbances. Moreover, this result was extended to the one for linear time-delay systems with both disturbances and polytopic-type uncertainties. In addition, our results were less conservative than some previous ones. Two numerical examples have been given to demonstrate the effectiveness and benefits of our proposed results.

\section{References}

[1] S. P. Boyd, L. El Ghaoui, E. Feron, V. Balakrishnan, Linear matrix inequalities in system and control theory, SIAM, Philadelphia, 1994.

[2] E. A. Cross, I. M. Mitchell, Level set methods for computing reachable sets of systems with differential algebraic equation dynamics, in: American Control Conference, 2008, IEEE, 2008.

[3] Z. Feng, J. Lam, An improved result on reachable set estimation and synthesis of time-delay systems, Appl. Math. Comput. 249 (2014) $89-97$.

[4] Z. Feng, J. Lam, On reachable set estimation of singular systems, Automatica 52 (2015) 146-153.

[5] E. Fridman, U. Shaked, On reachable sets for linear systems with delay and bounded peak inputs, Automatica 39 (2003) $2005-2010$.

[6] K. Gu, J. Chen, V. L. Kharitonov, Stability of time-delay systems, Springer Science \& Business Media, 2003.

[7] Q.-L. Han, Improved stability criteria and controller design for linear neutral systems, Automatica 45 (2009) 1948-1952.

[8] Y. He, Q.-G. Wang, L. Xie, C. Lin, Further improvement of free-weighting matrices technique for systems with time-varying delay, IEEE Trans. Autom. Control 52 (2007) 293-299.

[9] L. V. Hien, H. M. Trinh, A new approach to state bounding for linear time-varying systems with delay and bounded disturbances, Automatica 50 (2014) $1735-1738$.

[10] H. Jack, Theory of functional differential equations, New York: Spring-Verlag, 1977.

[11] J.-H. Kim, Improved ellipsoidal bound of reachable sets for time-delayed linear systems with disturbances, Automatica 44 (2008) $2940-2943$.

[12] J.-H. Kim, Note on stability of linear systems with time-varying delay, Automatica 47 (2011) 2118-2121.

[13] O. Kwon, S.-M. Lee, J. H. Park, On the reachable set bounding of uncertain dynamic systems with time-varying delays and disturbances, Inform. Sci. 181 (2011) 3735-3748.

[14] O.-M. Kwon, M.-J. Park, S.-M. Lee, J. H. Park, E.-J. Cha, Stability for neural networks with time-varying delays via some new approaches, IEEE Trans. Neural Netw. Learn. Syst. 24 (2013) 181-193.

[15] J. Lam, B. Zhang, Y. Chen, S. Xu, Reachable set estimation for discrete-time linear systems with time delays, Int. J. Robust Nonlinear Control 25 (2015) 269-281.

[16] X. Liao, L. Wang, P. Yu, Stability of dynamical systems, Elsevier Press, Amsterdam, The Netherland, 2007.

[17] J. Maidens, M. Arcak, Reachability analysis of nonlinear systems using matrix measures, IEEE Trans. Autom. Control 60 (2015) $265-270$.

[18] I. M. Mitchell, A. M. Bayen, C. J. Tomlin, A time-dependent Hamilton-Jacobi formulation of reachable sets for continuous dynamic games, IEEE Trans. Autom. Control 50 (7) (2005) 947-957.

[19] P. T. Nam, P. N. Pathirana, Further result on reachable set bounding for linear uncertain polytopic systems with interval time-varying delays, Automatica 47 (2011) 1838-1841.

[20] P. Park, J. W. Ko, C. Jeong, Reciprocally convex approach to stability of systems with time-varying delays, Automatica 47 (2011) $235-238$.

[21] C. Shen, S. Zhong, The ellipsoidal bound of reachable sets for linear neutral systems with disturbances, J. Frankl. Inst. 348 (9) (2011) 2570-2585.

[22] Y. Shen, J. Wang, Almost sure exponential stability of recurrent neural networks with Markovian switching, IEEE Trans. Neural Netw. 20 (2009) 840-855.

[23] J. Sun, G. Liu, J. Chen, Delay-dependent stability and stabilization of neutral time-delay systems, Int. J. Robust Nonlinear Control 19 (12) (2009) 1364-1375.

[24] Z. Wang, Y. Wang, Y. Liu, Global synchronization for discrete-time stochastic complex networks with randomly occurred nonlinearities and mixed time delays, IEEE Trans. Neural Netw. 21 (2010) 11-25.

[25] M. Wu, Y. He, J.-H. She, G.-P. Liu, Delay-dependent criteria for robust stability of time-varying delay systems, Automatica 40 (8) (2004) $1435-1439$.

[26] B. Zhang, J. Lam, S. Xu, Reachable set estimation and controller design for distributed delay systems with bounded disturbances, J. Frankl. Inst. 351 (6) (2014) 3068-3088.

[27] B. Zhang, J. Lam, S. Xu, Relaxed results on reachable set estimation of time-delay systems with bounded peak inputs, Int. J. Robust Nonlinear ControlDoi:10.1002/rnc.3395.

[28] H. Zhang, Z. Liu, Stability analysis for linear delayed systems via an optimally dividing delay interval approach, Automatica 47 (2011) 2126-2129.

[29] Z. Zuo, Y. Chen, Y. Wang, D. W. Ho, M. Z. Chen, H. Li, A note on reachable set bounding for delayed systems with polytopic uncertainties, J. Frankl. Inst. 350 (7) (2013) 1827-1835.

[30] Z. Zuo, Y. Fu, Y. Wang, Results on reachable set estimation for linear systems with both discrete and distributed delays, IET Contr. Theory Appl. 6 (2012) 2346-2350. 
[31] Z. Zuo, D. W. Ho, Y. Wang, Reachable set bounding for delayed systems with polytopic uncertainties: the maximal Lyapunov-Krasovskii functional approach, Automatica 46 (2010) 949-952. 